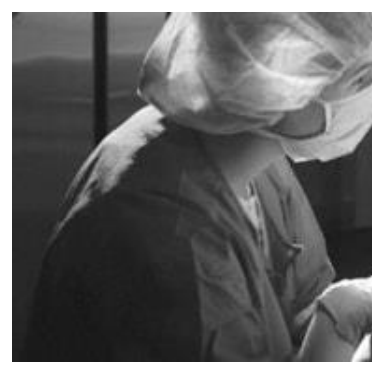

E-ISSN: 2616-3470

P-ISSN: 2616-3462

(C) Surgery Science

www.surgeryscience.com

2019; 3(3): 73-76

Received: 14-05-2019

Accepted: 16-06-2019

\section{Arshad Rashid}

(1) Surgeon Specialist, New City Hospital, Srinagar, Jammu and Kashmir, India

(2) Lecturer, Department of Surgery, Government Medical College \& Associated Hospitals, Srinagar, Jammu and Kashmir, India

Asim Rafiq Laharwal

Lecturer, Department of Surgery, Government Medical College \& Associated Hospitals, Srinagar, Jammu and Kashmir, India

Ajaz Ahmad Wani

Surgeon Specialist, New City

Hospital, Srinagar, Jammu and

Kashmir, India

\section{Correspondence}

Arshad Rashid

(1) Surgeon Specialist, New City Hospital, Srinagar, Jammu and Kashmir, India

(2) Lecturer, Department of Surgery, Government Medical College \& Associated Hospitals, Srinagar, Jammu and Kashmir, India

\section{International Journal of Surgery Science}

\section{Long term results of cyanoacrylate glue versus suture fixation for mesh in Lichtenstein hernia repair: A prospective randomized controlled trial}

\author{
Arshad Rashid, Asim Rafiq Laharwal and Ajaz Ahmad Wani
}

DOI: https://doi.org/10.33545/surgery.2019.v3.i3b.149

\section{Abstract}

Background: Controversies exist as to the best method of mesh fixation in Lichtenstein hernioplasty. The aim of this study was to compare cyanoacrylate glue fixation with suture fixation of mesh, with regards to recurrence rate and chronic groin pain.

Methods: This was a prospective randomized controlled study conducted in a perpheral hospital over a period of seven years. One hundred and fifty six patients of Lichtenstein hernia repair were included. Mesh was fixed by sutures and cyanoacrylate glue in 78 patients each. Only those patients were completed the follow up of 4 years were included in the final analysis.

Results: The mean operative time in suture fixation was $62.56 \pm 10.31$ versus $52.12 \pm 8.68$ minutes in glue fixation $(p<0.0001)$. The intra-operative complication rates did not differ significantly between the two techniques $(\mathrm{p}=0.7613)$. The post-operative pain scores were significantly lesser in the glue group [5.78 \pm 0.17 versus $6.71 \pm 0.29, p=0.0341]$. Among the suture fixation group four $(12.90 \%)$ patients had chronic groin pain, at the end of four-year follow up, as compared to three $(10.71 \%)$ patients in the glue group $(\mathrm{p}=$ 0.7342 ). The severity of chronic groin pain was also not found to be statistically different $[5.23 \pm 0.09$ in suture group versus $5.18 \pm 0.11$ in glue group, $\mathrm{p}=0.7954]$. The recurrence rates were similar between the two groups.

Conclusion: The frequency and severity of chronic groin pain, and recurrence rate do not depend on the method of mesh fixation.

Keywords: Cyanoacrylate glue, groin hernia, lichtenstein repair, inguinal hernia, chronic groin pain, recurrence

\section{Introduction}

To the contemporary surgeon, it would be unthinkable to expect a career without being competent enough in the performance of repair for inguinal hernias. The lifetime risk of groin hernia is $27-43 \%$ in men and $3-6 \%$ in women. Worldwide, more than 20 million people undergo inguinal hernia repair annually ${ }^{[1]}$. In majority of cases, surgical intervention is successful, however, recurrences necessitate reoperations in $10-15 \%$ of cases ${ }^{[2]}$. Another major long-term disability that occurs in about $10-12 \%$ of surgical repairs is chronic groin pain, which is defined as inguinal pain lasting for more than three months ${ }^{[3]}$.

The Lichtenstein repair with an onlay placement of mesh is the criterion standard for open inguinal hernia surgery ${ }^{[4]}$. Some of the complications of mesh fixation include: mesh migration, hernia recurrence, "meshoma" formation, chronic pain, and infection ${ }^{[5-7]}$. The various mesh fixation methods in open inguinal hernia repair are: staples, self-fixing, fibrin sealants, glues and sutures ${ }^{[5-8]}$. Controversies exist as to the best method of fixation. It has been suggested that using glue, in preference to suture, to fix the mesh may decrease the incidence of post-operative pain including chronic groin pain ${ }^{[8-10]}$. Most of the papers published on chronic groin pain include only a follow-up of 1 year ${ }^{[8-11]}$. With this thing in mind we conducted a study comparing cyanoacrylate glue (CG) fixation with suture fixation of mesh, in Lichtenstein hernioplasty, with a minimum follow-up of four years.

\section{Methods}

This study was a prospective randomized controlled trial comprising of a cohort of 156 patients, 78 each for suture fixation and CG fixation of mesh in Lichtenstein repair. 
A computer-generated chart did the randomization. All the repairs were done by a single surgeon (the corresponding author) with primary aim being comparison of recurrence and chronic groin pain in suture versus CG fixation of mesh in Lichtenstein repair. This study was conducted between March 2012 and March 2019 at a peripheral hospital of Kashmir. Patients were operated during the first three years till March 2015.

All of the cases were admitted on an elective basis and only the uncomplicated hernias were taken up for the study. Patients with bilateral and recurrent hernias were excluded from the study along with females, children less than 16 years of age and those with contraindications for general anaesthesia. All of the patients were advised to empty their urinary bladder just before surgery. Lichtenstein repair was done in the standard fashion. In one group, interrupted 2-0 polypropylene sutures (Prolene ${ }^{\circ}$; Ethicon by Johnson \& Johnson India Pvt. Ltd., Mumbai, IN) were used to fix the mesh to the pubic tubercle, inguinal ligament and the conjoint tendon, while as in the other group, CG (Netacryl@; Dr Reddy's Laboratories Ltd., Hyderabad, IN) was used to fix the mesh at these sites.

Various intraoperative and postoperative parameters like operative time, complications, postoperative pain, postoperative stay, time to return to activities of daily life and recurrence rates were evaluated. The operative time was defined as the time taken from incising the skin to the time of suturing/stapling of skin. Postoperative pain was measured by visual analog scale (VAS). The pain scores were obtained at 1, 2, 4, 6, 8, 12 and 24 hours postoperatively. Analgesics were administered if the VAS score was $>6$. Wound was inspected for any soakage or signs of infection. The follow up was initially done every fortnightly for two months and thereafter monthly for the first year and then every three months for the next three years for a total follow up of four years and the time taken for return to routine work and any recurrence was noted. Patients were also interviewed for chronic groin pain on follow up, which was defined as pain in the inguinal region persisting beyond 3 months. If chronic groin was present, VAS score was used to assess its severity.

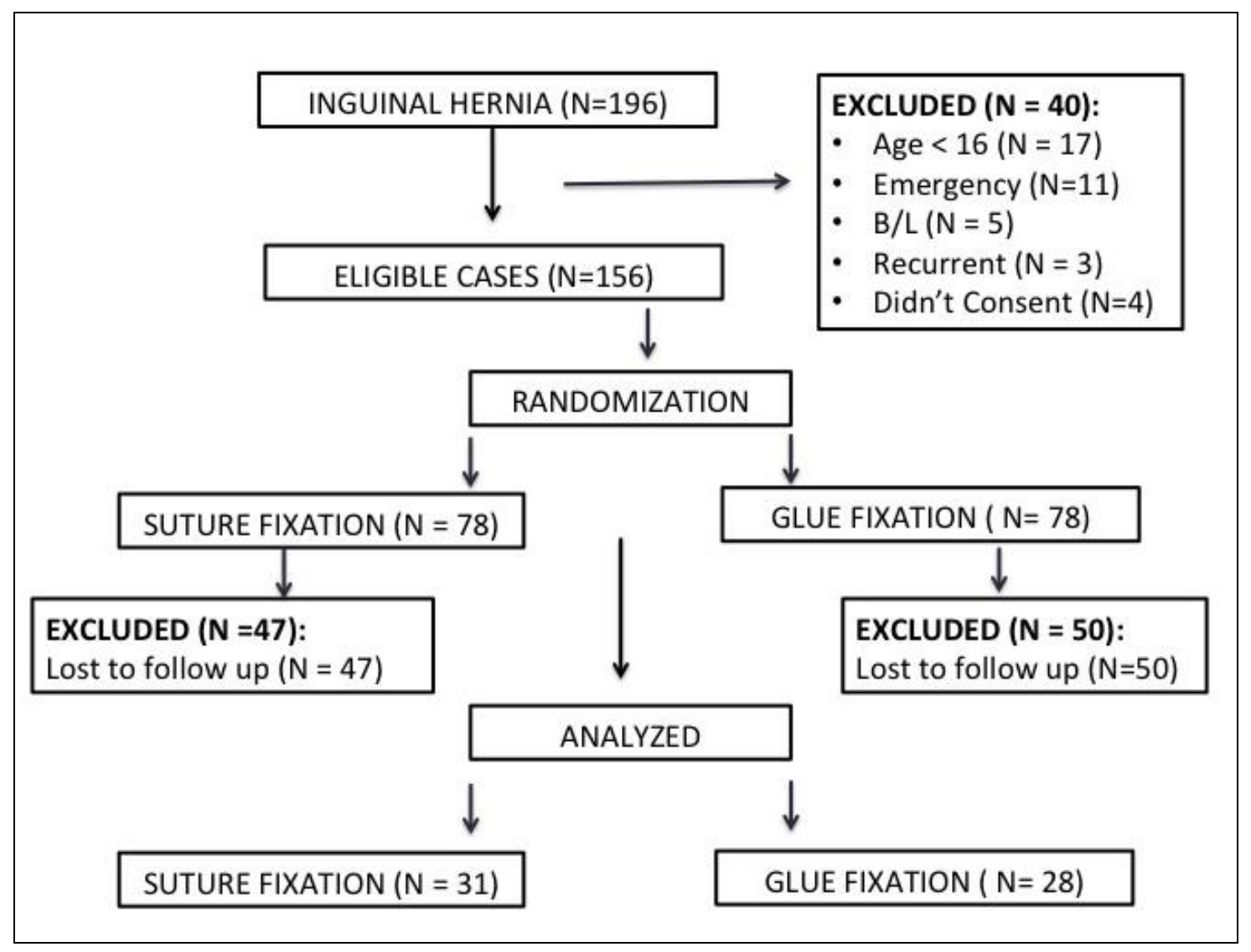

Fig 1: The CONSORT flow diagram of the study.

An approval from the institutional ethical committee was obtained for the purpose of this study. A written and informed consent was taken from the patients for publication and analysis of their data after explaining to them the protocol of the study in their own language. This clinical trial was registered with the local trials registry (CT-11/2012). The data thus collected was compiled and analyzed using SPSS version 22 for Mac (IBM Corporation, New York, 2012). To calculate the P-value, Fisher's exact test and Pearson's chi-square test were applied to compare the frequencies for categorical parameters, and the unpaired t-test was used to compare the means (2-tailed) among continuous variables. The results were calculated on $95 \%$ confidence interval. A P-value $<0.05$ was considered significant.

\section{Results}

During the study period a total of 196 inguinal hernia patients were treated, out of which 40 were excluded as they did not meet the critera of study protocol. The reamining 156 patients, who underwent Lichtenstein repair, were offered mesh fixation by $\mathrm{CG}$ or suture in equal numbers. In the suture fixation group, 47 patients were lost to follow-up and were not included for final analysis. In the CG fixation group, 50 patients were lost to follow up and were not included for final analysis. Thus a total of 31 patients of suture fixation and 28 patients of CG fixation were included in the final analysis [Figure 1].

The baseline parameters were similar in both the groups and all efforts were made to minimize the confounding factors [Table 1]. The mean operative time was significantly increased in the 
suture fixation group as compared to the $\mathrm{CG}$ fixation group $(62.56 \pm 10.31$ versus $52.12 \pm 8.68$ minutes, $p<0.0001)$. The total number of intra-operative complications were similar in the two groups [1 $(3.22 \%)$ versus $1(3.57 \%), p=0.7648]$. Bleeding was encountered in one patients in each group $(\mathrm{p}=0.7648)$, and was easily controlled by local methods and did not need any transfusion. There were no other intra-operative complications noted in either of the groups.

The mean postoperative pain scores (VAS) were significantly lower in the CG group $[5.78 \pm 0.17$ versus $6.71 \pm 0.29, \mathrm{p}=$ 0.0341]. The total number of post-operative complications was similar in both the groups. The various post-operative variables are given in Table 2 .

Table 1: Baseline Parameters of the two groups

\begin{tabular}{|c|c|c|c|c|}
\hline \multicolumn{2}{|c|}{ Parameter } & Suture Fixation $(\mathrm{N}=31)$ & Glue Fixation $(\mathrm{N}=28)$ & P Value \\
\hline \multicolumn{2}{|c|}{ Mean Age (Years) } & $52.34 \pm 9.42$ & $56.72 \pm 8.35$ & 0.7821 \\
\hline \multicolumn{2}{|c|}{ Mean BMI $\left(\mathrm{Kg} / \mathrm{m}^{2}\right)$} & $23.45 \pm 0.21$ & $23.12 \pm 0.19$ & 0.9665 \\
\hline \multirow{3}{*}{ EHS Type (N) } & Medial & $12(38.70 \%)$ & $10(35.72 \%)$ & 0.3132 \\
\hline & Lateral & $19(61.29 \%)$ & $18(64.28 \%)$ & 0.7954 \\
\hline & Femoral & 0 & 0 & - \\
\hline \multirow{3}{*}{ Defect Size $(\mathrm{N})$} & $\mathrm{I}(<1.5 \mathrm{~cm})$ & $8(25.80 \%)$ & $8(28.57 \%)$ & 0.9231 \\
\hline & II $(1.5-3 \mathrm{~cm})$ & $18(58.06 \%)$ & $17(60.71 \%)$ & 0.8986 \\
\hline & III $(>3 \mathrm{~cm})$ & $5(16.12 \%)$ & $3(10.71 \%)$ & 0.5324 \\
\hline
\end{tabular}

The recurrence rate at four-year follow up was not statistically different in suture group as compared to the $\mathrm{CG}$ group [1 $(3.22 \%)$ versus $1(3.57 \%), p=0.7648]$. Among the suture fixation group four $(12.90 \%)$ patients had chronic groin pain, at the end of four-year follow up, as compared to three $(10.71 \%)$ patients in the CG group ( $\mathrm{p}=0.7342$ ). The severity of chronic groin pain was also not found to be statistically different in the two groups at the end of four years of follow up [5.23 \pm 0.09 in suture group versus $5.18 \pm 0.11$ in CG group, $\mathrm{p}=0.795 \overline{4}]$.

\section{Discussion}

Inguinal hernia repair is one of the most commonly performed surgeries in the world. Lichtenstein hernioplasty has Grade ' $A$ ' recommendation for primary unilateral uncomplicated hernias as per the European Hernia Society guidelines [12]. The mesh fixation in Lichtenstein repair can be accomplished in many ways ${ }^{[5-8]}$. Synthetic mesh fixation in hernia repair involves a consideration of the strength of fixation versus the risk of trauma to local tissues. The method of fixation of the mesh is one of the factors that have been blamed for postoperative pain. It has been postulated that sutures, mostly non-absorbable, can increase pain by nerve entrapment or foreign body reaction and non-traumatic methods of fixation produce less pain without compromising the outcome ${ }^{[13]}$.

Consensus does not exist about the "best fixation method" and most of the decisions on fixation depend upon individual preference ${ }^{[3]}$. Any evidence that reports on a particular fixation method improving various outcome measures may have a significant impact on clinical practice. The current paper reports on the comparison of method of mesh fixation (CG versus suture) in Lichtenstein repair in a homogenous population over a three-year period with an analysis after a follow-up of four years at a district level hospital. To the best of our knowledge, this is the first study in English literature that reports on the severity and incidence of chronic groin pain at four years after Lichtenstein hernioplasty.

The method of mesh fixation was not found to have any significant influence on the intraoperative complication rate or recurrence rate or chronic groin pain rate. In the current study, we found that the operative time was lower with glue as compared to the suture fixing method, as consistent with all the studies. Pain in the immediate post-operative period, is also significantly lower in the glue mesh fixation patients. Although long-term recurrence rates have not been determined and large hernias often have been excluded, none of the randomized controlled studies and meta-analyses conducted till date have reported any significant difference in the recurrence rate in suture versus glue fixation of mesh in Lichtenstein hernioplasty [3, 12-16]. Even with a fairly long follow-up of four-years, the results of our study are consistent with these reports.

Table 2: Post-operative variables of the two groups

\begin{tabular}{|c|c|c|c|c|}
\hline \multicolumn{2}{|c|}{ Parameter } & Suture Fixation $(\mathbf{N}=\mathbf{3 1})$ & Glue Fixation $(\mathrm{N}=28)$ & P Value \\
\hline \multicolumn{2}{|c|}{ Mean Pain Scores (VAS) } & $6.71 \pm 0.29$ & $5.78 \pm 0.17$ & 0.0341 \\
\hline \multicolumn{2}{|c|}{ Mean Postop Stay (Days) } & $1.42 \pm 0.37$ & $1.12 \pm 0.11$ & 0.0425 \\
\hline \multirow{4}{*}{ Complications (N) } & Seroma & $1(3.22 \%)$ & $1(3.57 \%)$ & 0.7648 \\
\hline & Wound Infection & 0 & 0 & - \\
\hline & Testicular Swelling & $1(3.22 \%)$ & $1(3.57 \%)$ & 0.7648 \\
\hline & Hematoma formation & $1(3.22 \%)$ & $1(3.57 \%)$ & 0.7648 \\
\hline \multirow{2}{*}{\multicolumn{2}{|c|}{ Total Complications (N) }} & $3(9.67 \%)$ & $3(10.71 \%)$ & 0.7613 \\
\hline & Return to normal Activity (Days) & $15.23 \pm 1.31$ & $12.24 \pm 1.08$ & 0.0312 \\
\hline
\end{tabular}

Any pain persisting in the groin beyond a period of three months after surgery is known as chronic groin pain ${ }^{[3]}$. In our study we did not find any difference either in frequency or on the intensity of chronic groin pain at the end of four year follow up in the two groups. Most of the studies published till date have measured chronic pain incidence at 3 months, or at 6 months, and longest only at 1 year ${ }^{[14-22]}$. The combined chronic pain rates for mesh fixation of various types were: $14.7 \%$ for sutures, $7.6 \%$ for glue,
$3.7 \%$ for fibrin sealant, and $18.2 \%$ for self-fixing meshes ${ }^{[3]}$. We believe that with a longer follow-up, the chronic groin pain stabilizes and is independent of the method of fixation, but further studies are warranted to substantiate the facts.

The major causes of postoperative pain are the stretching of the wound during surgery and the length of the fascial incision [23]. As more of stretching occurs during suture fixation of mesh, it is but natural to expect that acute post-operative pain would be 
more in the suture fixation group, which was indeed the case. The maximum scores of pain were observed at 4 hours postoperatively in both the groups, presumably due to exhaustion of the effects of analgesia and anesthesia. Less acute postoperative pain translated into early ambulation, acceptance of orals, and discharge of patients in the CG group. This was also reflected in the earlier resumption of routine work by this group of patients. As most of the patients undergoing CG fixation were discharged within the first postoperative day [24 hour period], we believe that this method of fixation might be beneficial in surgeries planned on a day care basis.

The present study has some potential limitations. We acknowledge the fact that more than $50 \%$ of the study population was lost to follow-up, but, this reflects the fact that we strongly adhered to our study protocol and only included those participants who completed a follow-up of four years. The sample size was small and the population was homogenous, so extrapolation of results to other parts of the globe may not be valid. Our study was not a double-blinded one. However, the pain assessment was somewhat blinded as all scoring was performed by the attending nurse who was unaware of the ongoing study.

\section{Conclusion}

The results of the current study fail to document any definite advantage of glue fixation over suture fixation as regards the incidence and severity of chronic groin pain. Though the glue fixation method scores over suture fixation as regards to the operative time and acute post-operative pain. The recurrence rates are similar in both the methods of fixation.

\section{References}

1. Kingsnorth A, LeBlanc K. Hernias: inguinal and incisional. Lancet. 2003; 362(9395):1561-1571.

2. Kockerling F, Simons MP. Current concepts of inguinal hernia repair. Visc Med. 2018; 34(2):145-150.

3. The HerniaS urge Group. International guidelines for groin hernia management. Hernia. 2018; 22(1):1-165.

4. Lichtenstein IL, Shulman AG, Amid PK, Montilor M. The tension-free hernioplasty. Am J Surg. 1989; 157(2):188193.

5. Schwab R, Schumacher O, Junge K, Binnebosel M, Klinge U, Schumpelick V. Fibrin sealant for mesh fixation in Lichtenstein repair: biomechanical analysis of different techniques. Hernia. 2007; 11(2):139-145.

6. Amid PK. Radiologic images of meshoma. A new phenomenon causing chronic pain after prosthetic repair of abdominal wall hernias. Arch Surg. 2014; 139(12):12971298.

7. Kumar S, Wilson RG, Nixon SJ, Macintyre IMC. Chronic pain after laparoscopic and open mesh repair of groin hernia. Br J Surg. 2002; 89(11):1476-1479.

8. de Goede B, Klitsie PJ, van Kempen BJH et al. Metaanalysis of glue versus sutured mesh fixation for Lichtenstein inguinal hernia repair. Br J Surg. 2013; 100(6):735-742.

9. Ladwa N, Sajid MS, Sains P, Baig MK. Suture mesh fixation versus glue mesh fixation in open inguinal hernia repair: a systematic review and meta-analysis. Int J Surg. 2013; 11(2):128-135.

10. Colvin HS, Rao A, Cavali M, Campanelli G, Amin AI. Glue versus suture fixation of mesh during open repair of inguinal hernias: a systematic review and metaanalysis. World J Surg. 2013; 37(10):2282-2292.
11. Sanders DL, Waydia S. A systematic review of randomized control trials assessing mesh fixation in open inguinal hernia repair. Hernia. 2014; 18:165-176.

12. Miserez M, Peeters E, Aufenacker T et al. Update with level 1 studies of the European Hernia Society guidelines on the treatment of inguinal hernia in adult patients. Hernia. 2014; 18:151-163.

13. Jeyakumar S, Chitrambalam TG, Chandrasekaran S. Glue versus suture for mesh fixation in open inguinal hernia repair. Int Surg J. 2018; 5(4):1443-48.

14. Hidalgo M, Castillo MJ, Eymar JL, Hidalgo A. Lichtenstein inguinal hernioplasty: sutures versus glue. Hernia. 2005; 9(3):242-244.

15. Kim-Fuchs C, Angst E, Vorburger S, Helbling C, Candinas D, Schlumpf R. Prospective randomized trial comparing sutured with sutureless mesh fixation for Lichtenstein hernia repair: long-term results. Hernia. 2012; 16:21-27.

16. Nowobilski W, Dobosz M, Wojciechowicz T, Mionskowska L. Lichtenstein inguinal hernioplasty using butyl-2-cyanoacrylate versus sutures: preliminary experience of a prospective randomized trial. Eur Surg Res. 2004; 36:367-370.

17. Shen YM, Sun WB, Chen J, Liu SJ, Wang MG. NBCA medical adhesive (n-butyl-2-cyanoacrylate) versus suture for patch fixation in Lichtenstein inguinal herniorrhaphy: a randomized controlled trial. Surgery. 2012; 151:550-555.

18. Wong JU, Leung TH, Huang CC, Huang CS. Comparing chronic pain between fibrin sealant and suture fixation for bilayer polypropylene mesh inguinal hernioplasty: a randomized clinical trial. Am J Surg. 2011; 202:34-38.

19. Paajanen H, Kossi J, Silvasti S, Hulmi T, Hakala T. Randomized clinical trial of tissue glue versus absorbable sutures for mesh fixation in local anaesthetic Lichtenstein hernia repair. Br J Surg. 2011; 98:1245-1251.

20. Paajanen H. Do absorbable mesh sutures cause less chronic pain than nonabsorbable sutures after Lichtenstein inguinal herniorrhaphy? Hernia. 2002; 6:26-28.

21. Lionetti R, Neola B, Dilillo S, Bruzzese D, Ferulano GP. Sutureless hernioplasty with light-weight mesh and Wbrin glue versus Lichtenstein procedure: a comparison of outcomes focusing on chronic postoperative pain. Hernia. 2012; 16:127-131.

22. Pierides G, Scheinin T, Remes V, Hermunen K, Vironen J. Randomized comparison of self-fixating and sutured mesh in open inguinal hernia repair. Br J Surg. 2012; 99:630-636.

23. Rashid A, Nazir S, Kakroo SM, Chalkoo MA, Razvi SA, Wani AA. Laparoscopic interval appendectomy versus open interval appendectomy: A prospective randomized controlled trial. Surg Laparosc Endosc Percutan Tech. 2013; 23(1):93-96. 